\title{
La rationalisation de l'éducation physique en France, 1950-1966 : la pédagogie du « rendement et du contrôle »
}

\author{
Philippe Sarremejane
}

\begin{abstract}
Dans les années d'après-guerre (1950-1966), l'éducation physique a connu, en France, un fort mouvement de rationalisation méthodologique. Mue par une quête de reconnaissance sociale et le désir d'avoir le statut d'une véritable discipline scolaire, l'éducation physique cherche à produire une forme de "rendement» associé à une procédure de contrôle des «acquis ». Il s'agit bien de mesurer les " effets » de l'enseignement, le progrès. Les outils d'évaluation n'étaient alors, ni performants, ni unifiés, ni généralisés. La démarche novatrice commencera par une estimation de la capacité physique des élèves afin de pouvoir définir des groupes homogènes d'élèves. L'individualisation est la méthode la plus efficace. Progressivement, les outils permettant d'estimer ce niveau de départ vont devenir de plus en plus performants et l'élaboration de la Table de cotation de Jean Letessier sera le moment culminant de cette innovation. Cette nouvelle méthodologie présente des modes de rationalité théoriques et pratiques substantiels. Toutefois, le contrôle du progrès va s'avérer difficile. Visiblement les conditions scolaires de la pratique ne permettent pas de faire la preuve que les élèves ont véritablement progressé pendant le cours d'éducation physique. De nombreuses tensions demeurent.
\end{abstract}

In the post-war period (1950-1966), physical education in France went through a strong movement of methodological rationalization. Driven by a search for social acceptance and a desire to be recognized as a fundamental school subject, physical education sought to produce a form of efficiency combined with a procedure for the control of learning. The goal was to measure the effects of teaching, that is, the "progress" made. Yet during this period evaluation tools were neither effective nor unified or generalized. The innovative process begins with an estimation of the physical capacities of the pupils so as to define comparable level groups. Childcentredness becomes the most efficient method. Gradually, the tools allowing us to estimate this level become more impressive; the development of the classification table of Jean Letessier can be seen as the height of this innovation. This new methodology presents important theoretical and practical modes of rationality. However, controlling "progress" will turn out to be challenging. Clearly, the school conditions of teaching make it difficult to prove that the pupils have really progressed in their physical education classes. Many tensions remain.

Au début des années 1950, les théoriciens de la discipline éducation physique portent un intérêt tout particulier à la valeur physique. Cet intérêt est associé à la volonté de rationaliser la pratique d'enseignement en contrôlant ses effets. L'éducateur se doit de mettre 
en place une procédure d'intervention efficace tout simplement parce que « l'enfant vient à l'école pour se perfectionner »'. L'éducateur est responsable de la transmission, il est responsable des progrès de l'élève. C'est ce moment, cette émergence de l'esprit de rendement, qui a fortement marqué la discipline dont nous voulons rendre compte.

Cette étude se fonde sur un double corpus : la revue Education physique et sport (EPS) et les ouvrages de référence des auteurs qui ont publié dans la revue. La revue EPS représente en France, depuis 1950, la culture professionnelle des professeurs d'éducation physique. Au début des années 50, la revue fait appel à la publication, elle propose à des rédacteurs éventuels dix thèmes - (1) le sport, phénomène social, (2) le sport dans l'enseignement supérieur, (3) le sport et le milieu ouvrier, (4) le gymnase, (5) l'appréciation de la valeur physique, (6) l'organisation du travail de l'éducateur physique, (7) la compétition, (8) le rythme, (9) l'adaptation des méthodes étrangères à l'éducation physique française, (10) la nécessité et le rôle des éducateurs et des chercheurs. Le groupe de réflexion sur le thème de la valeur physique, groupe dit $\mathrm{n}^{\circ} 5$, sera le plus productif ${ }^{2}$. Nous avons retenu l'ensemble des articles de ce groupe portant sur la période 1950-1966. Pierre Parazols et Jean Letessier, tous deux professeurs d'éducation physique, seront les membres les plus influents du groupe. Ils sont les signataires majeurs de ces articles.

Notre travail, qui s'inscrit dans une perspective historique, a pour but d'apprécier la validité des modes de rationalisation annoncés. Sans nier le rôle des déterminants institutionnels ${ }^{3}$ et culturels plus larges, nous avons pris le parti de rester centré sur l'évolution des instruments de «contrôle ». Nous évoquerons aussi les conditions de faisabilité de la "pédagogie du rendement». Bien que la table de cotation Letessier, destinée à établir ce contrôle, fût largement diffusée, de nombreuses tensions demeurent quant à sa finalité première : évaluer les progrès des élèves.

"Rationaliser » s'entend ici comme la mise en place du contrôlable. L'intention n'est certainement pas neuve. Pourtant, elle s'exprime avec force pendant deux décennies. Le contrôle des effets de la pratique d'enseignement est une revendication insistante associée à l'image que la discipline entend donner d'elle-même.

1 Pierre Parazols, « Le contrôle en éducation physique », Education physique et sport, $\mathrm{n}^{\circ} 48(1960): 15$.

2 Cf. Le comité de rédaction, « Nos problèmes », Education physique et sport, ${ }^{\circ} 9$ (1952) : 47.

3 Que nous abordons uniquement de manière incidente. 
« Rationaliser » consiste à dégager des principes pratiques cohérents qui s'organisent en trois temps : contrôler avant l'enseignement la valeur physique de l'élève, la développer par l'enseignement, ce sera le perfectionnement, puis, enfin, évaluer le progrès après l'enseignement. Le moyen de cette rationalisation sera la production d'un instrument d'estimation de la valeur physique. Non seulement la procédure pédagogique doit être efficace mais aussi avoir un certain rendement ${ }^{4}$. La rigueur de la démarche doit permettre à l'enseignant d'avoir un repère de départ clairement établi et tangible afin de pouvoir se donner un but et l'atteindre. Ce plan de construction de l'enseignement sera, par analogie, comparable à un repérage dans l'espace. Pour savoir où l'on va, il faut d'abord savoir où l'on est et même savoir d'où l'on vient : " une action cohérente des éducateurs sera mieux à même de savoir d'où ils viennent et où ils vont $»^{5}$. L'évaluation est instrumentalisée, elle n'est plus seulement un moyen de donner des notes aux différentes compositions trimestrielles. Elle devient un moyen pédagogique.

Au-delà de la certification et de la production de notes, dont l'intérêt est indéniable, l'appréciation juste de la valeur physique apparait comme un moyen de " tri $"^{6}$ afin de faire des groupes homogènes. L'évaluation est aussi l'élément fondamental d'une unité méthodologique?.

D'abord, cerner ce sur quoi portera l'enseignement, puis élaborer un outil d'évaluation qui permettra de différencier les élèves et aussi d'établir le constat d'un progrès. Projet de connaissance d'abord,

4 «L'éducation physique, en ce qu'elle est au premier chef action, se doit d'obtenir un certain rendement ", Pierre Parazols , "L'estimation des capacités physiques », Education physique et sport, $\mathrm{n}^{\circ} 22$ (1954) : 11.

5 Pierre Parazols, «Méthode nouvelle ou nouvelles méthodes », Education physique et sport, $\mathrm{n}^{\circ} 44$ (1959) : 16 .

6 Contre la logique du groupement chronologique des élèves « L'éducateur physique [...] s'est vu conduit à faire un tri ... Il a découvert peu à peu combien le décalage est grand entre les capacités des divers sujets de sa classe. Il en est venu à individualiser son enseignement ». Pierre Parazols, "Le contrôle en Education physique », Education physique et sport, $\mathrm{n}^{\circ} 48(1960): 15$.

7 Ce problème de la recherche de l'unité de la méthode est essentiel durant cette période. Car après l'éclectisme instauré par les instructions officielles de 1945, les acteurs de la communauté et les institutionnels essaieront dans un premier temps de trouver l'unité de méthode plus par un cadrage temporel de la leçon (partie construite, partie fonctionnelle) que par une véritable unité des contenus. La gymnastique suédoise, l'hébertisme et l'initiation sportive persistent en filigrane derrière le " construit » et le « fonctionnel ». Les rapports complexes de l'éducation physique avec le sport, qui est devenu dès les années 50 un enjeu social et politique fondamental, s'exprimeront de manière tronquée dans les instructions de 1959, dont le caractère quasi anachronique sera réduit par les textes de 1962 et de 1967 qui feront de la pratique sportive l'essentiel des contenus d'enseignement de l'EPS. 
finalité pratique ensuite qui consiste surtout à optimaliser l'acte d'enseignement. Cette double rationalisation - théorique et pratiquea-t-elle abouti, cette instrumentalisation de la pratique a-t-elle obtenu des résultats tangibles? C'est ce que notre étude va essayer de dégager.

\section{Les moyens de l'appréciation de la valeur physique Les normes de rationalité de la mesure}

"S'il n'y a de science que du mesurable, les performances athlétiques apportent la condition sine qua non pour entreprendre une étude méthodique des qualités physiques $»^{8}$. Cette phrase d'inspiration aristotélicienne est à la fois méthodologique et programmatique. Méthodologique, car l'intention est bien d'opter pour une démarche de rationalisation, et programmatique puisqu'il s'agit " d'entreprendre ", c'est-à-dire de construire un outil d'évaluation des qualités physiques. Lorsqu'il écrit cela en 1966, Jean Letessier a pourtant déjà produit un tel outil. Il ne reste plus qu’à convaincre de l'utilité de sa table de cotation. C'est le problème de la généralisation de la table à l'ensemble de la corporation des enseignants d'éducation physique qui motive son discours.

C'est donc plus tôt, au début des années 50, que cette ambition scientifique de mesurer apparait. La mesure est un acte de rationalisation parce qu'elle impose une constante à une chose, parce qu'elle impose une grandeur constante prise comme référence à un état de chose indéterminé. De façon plus précise, mesurer, c'est attribuer des nombres aux choses, c'est établir une correspondance entre certaines propriétés des nombres et certaines propriétés des choses. C'est donc supposer que les propriétés fonctionnelles des nombres et celles des choses sont analogues, seule cette supposition permettant la substitution. Rappelons que la mesure a surtout une fonction pratique au sens où elle facilite l'action sur les choses. Car il est plus facile d'agir et de manipuler intellectuellement les nombres que ce qui leur correspond dans les choses.

Si la mesure consiste à établir un ordre, si son emploi est ressenti comme de plus en plus urgent, c'est que la pratique telle qu'elle est faite dans les années 50 ne présente pas suffisamment de cohérence. L'emploi d'une même norme de mesure par un groupe ou une corporation présente une finalité pratique certes, mais surtout sociale. La communauté des professeurs d'éducation physique ne sera une

8 Jean Letessier, « Table générale de cotation des performances athlétiques et barèmes pour les examens sportifs », Education physique et sport, $n^{\circ} 79$ (1966) : 18. 
véritable communauté que par l'emploi des mêmes référents théoriques et pratiques. Le lien communautaire assuré par l'ancrage institutionnel, qui définit au moyen de textes prescriptifs les finalités et les contenus disciplinaires, n'est pas suffisant. Il faut aussi, selon G. Colo, partager les mêmes outils pratiques, il faut partager surtout les mêmes instruments de contrôle des effets de la pratique, les instruments de mesure. Ainsi, écrit-il dans la revue EPS :

«Et pourtant, il est un remède bien simple : normaliser ! Faire pratiquer l'éducation physique sans mesurer les aptitudes et contrôler ces résultats, c'est travailler dans la nuit. Mais si pour contrôler ces résultats, il est imposé autant de barèmes de cotation et autant d'épreuves différentes qu'il est proposé d'examens à préparer et subir, c'est rendre la nuit plus noire encore. Un Educateur Physique répugne à travailler dans le désordre. Le mètre et le chronomètre le forgent net, loyal et objectif. Éducation Physique aux examens ? Crise de l'athlétisme ? Prestige légitime d'une corporation gagnant son pain à la sueur de son front ? Désir d'être pris au sérieux ? D'être entendus? - Entendons nous d'abord pour être entendus »'.

Le constat de la disparité des méthodes et des moyens de contrôle est dans l'après-guerre manifeste. L'unité disciplinaire est affaiblie. Les moyens de l'efficacité passent par des référents stables; certains enseignants s'emploient à les construire. Les expériences d'élaboration de barèmes ne sont pas rares même si elles restent isolées. Elles ne sont pas encore en mesure d'être unifiées et généralisées. Les barèmes sont produits au sein des établissements, au mieux pour un usage interne et collectif, au pire pour un usage strictement personnel. Le propos de Henri Talbot, Inspecteur Général, est dans ce sens édifiant : «On s'est beaucoup plaint de la diversité des barèmes de cotation des performances physiques, en particulier au moment des examens ou concours. Il n'y a pas d'instructions précises et générales à ce sujet et celles que l'on possède représentent, pour tel ou tel examen et pour lui seulement, une traduction immédiate des performances réalisées en notes de valeur, sur 20 par exemple. D'autre part, elles donnent prise à des sérieuses critiques quant à l'allure même de cette échelle de notes ${ }^{10}$.

9 G. Colo, « Normalisation », Education physique et sport, $n^{\circ} 28$ (1955) : 44.

10 Henri Talbot, « Propos introductif à l'article de Jean Letessier, Les performances sportives et leurs classements », Education physique et sport, n 29 (1956) : 3. 
Outre la regrettable absence d'un barème unique et équitable, c'est aussi la façon très subjective qu'ont les professeurs d'estimer leurs élèves qui est critiquée. L'illusion de "la sûreté du coup d'œil » est vivement dénoncée ${ }^{11}$. Contre cette approche très empirique de l'estimation, il faut désormais opter pour la médiation quantitative. Cette médiation interpose entre le sujet et le donné un référent stable : le nombre appliqué à une valeur constante. «Si vous pouvez mesurer ce dont vous parlez et l'exprimer par un nombre, vous savez quelque chose de votre sujet, sinon vos connaissances sont d'une pauvre espèce et bien peu satisfaisantes, quelle que soit la question dont vous vous occupiez ». Cette citation de Lord Kelvin est donnée en 1952 par Pierre Parazols ${ }^{12}$, qui sera le fervent promoteur de ce changement des mentalités. Le nombre fait exister la chose, il n'y a de connaissance véritable que du dénombrable ${ }^{13}$. Cette forme d'idéalisme mathématique doit être tempérée dans ce contexte par un souci pratique, par une exigence pratique : l'efficacité de l'intervention. « Et viser l'efficience, c'est tôt ou tard être conduit à la mesure $»^{14}$. C'est le désir de mieux faire, de mieux enseigner en l'occurrence, qui justifie l'exigence de rationalité et donc de la mesure.

\section{0-1966. La progression rationnelle des outils d'évaluation : du qualitatif au quantitatif}

Au début des années 1950, la notation de l'éducation physique lors de la composition de fin de trimestre prend trois formes, souvent associées : les épreuves isolées de type athlétique, les parcoursépreuves et les enchaînements gymniques. Le souci nouveau est de reprendre ces outils et de les améliorer pour qu'ils deviennent performants en tant qu'instruments de contrôle de la procédure d'enseignement. La composition d'éducation physique, soutient-on, ne tient pas compte du progrès de l'élève et donc de l'effet réel de l'enseignement, elle est une évaluation de type sommatif à l'issue du

11 «Certains ne veulent devoir ce portrait qu’à leur talent. Ils comptent sur la sûreté de leur coup d'œil, sur l'infaillibilité de leur jugement, sur la richesse de leur expérience ». Pierre Parazols, « Le contrôle en éducation physique », Education physique et sport, $\mathrm{n}^{\circ} 48$ (1960): 16 .

12 Pierre Parazols, « L'utilisation des installations », Education physique et sport, $\mathrm{n}^{\circ} 13$ (1952) : 33 .

13 Cette thèse à la fois gnoséologique et ontologique qui fait du nombre la chose même est ancienne. Elle est déjà présente chez les présocratiques et surtout chez Platon. Cf, le livre VII de La République.

14 Pierre Parazols, « Les groupes homogènes ", Education physique et sport, $\mathrm{n}^{\circ} 27$ (1955) : 11. 
trimestre. L'évaluation du progrès désormais perçue comme souci majeur nécessite de cibler la nature même de ce qui est l'objet des transformations et aussi de définir un outil fiable d'évaluation. L'intention première est de concilier précision de la mesure et estimation de la valeur physique générale voire de la personnalité de l'élève d'un point de vue global, ce que Hébert appelait « l'homme complet». L'estimation du " global » est tout à fait recevable dans son principe puisqu'il s'agit de donner l'image motrice la plus complète possible de l'élève. Mais très vite, les membres du groupe $\mathrm{n}^{\circ} 5$ s'aperçoivent que globalité et objectivité ne vont pas ensemble. L'exigence de rigueur des épreuves-tests avant l'enseignement ne pourra reprendre les critères qualitatifs, appréciés et subjectifs qui, souvent, entrent en ligne de compte dans les notes de composition de fin de trimestre :

«Il nous semble intéressant de bien distinguer la note de valeur physique, d'un emploi général, basée sur les cinq épreuves retenues : deux courses (vitesse et résistance), un saut (hauteur), un lancer (poids), un grimper (deux fois trois mètres chronométrés) et une appréciation plus large, adaptée aux exigences de l'établissement, et tenant compte des élèments tels qu’assiduité, tenue, comportement $»^{15}$.

On peut constater qu'en l'espace d'une quinzaine d'années les instruments de contrôle diagnostique gagnent progressivement en objectivité, en simplicité et en efficacité. Le qualitatif et l'approximatif laisseront progressivement la place à des modalités polycritériées fournies par des outils quantifiés, précis et efficaces.

L'évaluation initiale des capacités est faite au début par différents moyens qui très vite deviennent concurrents : le parcours-épreuve mono-critérié parfois associé à des échelles qualitatives, les parcoursépreuves polycritériés, les épreuves athlétiques isolées et enfin les épreuves combinées des compositions de fin de trimestre.

\section{La persistance de l'hébertisme : l'évaluation de la valeur physique par le parcours}

Dans l'après-guerre, le parcours Hébert perdure, vraisemblablement jusqu'à la fin des années 60 . Il est souvent le

15 Pierre Parazols, «Le contrôle en éducation physique », Education physique et sport, $n^{\circ} 48$ (1960) : 16. 
contenu même de la leçon de plein air. Puisque le maitre a des épigones fidèles, revenons brièvement à lui. En 1911, Georges Hébert publiait le Code de la force ${ }^{16}$. Le chapitre IX est consacré au «Parcoursépreuve ». Le parcours-épreuve peut éventuellement servir à mesurer la valeur physique. L'idéologie utilitariste de l'éducation physique selon Hébert retient surtout la capacité d'adaptation comme qualité fondamentale de la valeur physique, ce qu'il appelle «l'habileté à se débrouiller ». La variété des épreuves et le caractère imprévu du parcours concourent à solliciter ce " vrai critérium de la résistance » qu'est la durée d'accommodation ou d'adaptation à un nouveau travail $^{17}$. Autrement dit, l'imprévisibilité sera le caractère d'un véritable parcours puisqu'il est celui du terrain libre que l'on découvre en plein nature. L'adaptabilité, qui a pour corrélat l'imprévu, ne facilite pas l'élaboration d'un parcours dont le caractère normatif et stable devrait servir de gage d'objectivité à une procédure d'évaluation. L'évaluation ne sera possible que sur un parcours aménagé du type «parcours tracé et repéré ${ }^{18}$. Quant au critère, Hébert préconise le temps pour évaluer la valeur physique générale :

«Si l'on désire avoir une véritable mesure de la valeur physique générale au moyen de l' 'épreuve-parcours ', il est nécessaire d'établir une échelle de performances cotées en temps. Pour que cette échelle soit suffisamment juste, il faut, sur un parcours bien établi, effectuer de nombreux essais de performances avec des sujets dont on connaît déjà la valeur personnelle, par le procédé des épreuves mesurables de la série-type ... On peut alors, d'après les performances accomplies par ces sujets sur le dit parcours, déduire des cotes en temps et déterminer ainsi des niveaux d'aptitudes : insuffisant, moyen, supérieur, athlétique $»^{19}$.

Dans l'après-guerre, la thématique de "l'estimation de la valeur physique » trouve dans le parcours-épreuve un outil d'évaluation conforme à la doctrine du maitre. Le parcours est le modèle type de la phase d'évaluation initiale avant la constitution des groupes. En 1950, Parazols réalise ses groupes de valeur physique à l'issue d'un parcours-épreuve. Il reprend littéralement la formule hébertiste, selon laquelle le parcours doit offrir une synthèse de tous les exercices.

Georges Hébert, Le code de la force (Paris : Vuibert, 1911).

Ibid., 145.

Ibid., 146.

Ibid., 143. 
Cette synthèse a bien sûr pour vocation de rendre compte du plus grand nombre de capacités.

Dans l'institution scolaire, le parcours est du type tracé et repéré, c'est un parcours d'obstables chronométré qui comporte aussi des épreuves (fig. 1). La distance exigée varie en fonction de l'âge des élèves. Les capacités sollicitées sont : la vitesse, la coordination, l'équilibre, la force, l'adresse et la résistance. Seul le temps chronométré rend compte de ce que " vaut » l'élève. Certaines épreuves contrarient le principe de rapidité d'exécution, car il faut aussi savoir maintenir son équilibre sur une poutre, être capable de projeter un lancer lourd dans une zone. Un ensemble de consignes codifie ces épreuves afin de reprendre le plus rapidement possible le parcours. En lancer, par exemple, on continue lorsque le premier poids tombe dans la zone la plus éloignée. Le principe temporel écrase rapidement l'expression des autres capacités. Contre cela, il sera proposé une formule moins réductrice : le parcours fractionné ${ }^{20}$. Ce mode propose des parcours plus courts avec un nombre restreint d'épreuves. Un parcours sollicitera la vitesse, l'adresse, l'agilité ; un autre, la détente, l'équilibre et la force. D'autre part, pour ne pas fausserl'expression des différentes habiletés, le chronomètre est arrêté avant certaines épreuves et redéclenché après.

Les adeptes des parcours-mesure sont délibérément opposés aux épreuves «isolées ». La valeur analytique du sujet ne peut être donnée que par le parcours. Pourtant, au début des années 60, pour gagner en efficacité dans la mesure, il devra être aménagé : " pour plus de précision, il semble utile de placer l'épreuve d'adresse après la vitesse, qui peut le plus peut le moins ... le grimper sera placé après une épreuve de 'relâchement' ... nous avons toujours placé le lancer lourd, épreuve de force, en fin de parcours $»^{21}$. L'important est ne pas trop vite épuiser l'élève, qui, lors des épreuves de fin de parcours, aurait des performances insignifiantes. Les éducateurs vont même plus loin : au sein du parcours, en plus du temps global, on évalue chacune des épreuves. Un barème de points adapté à chaque épreuve pondère le temps mis sur le parcours. Puis, pour être encore plus précis, on mesure toutes les épreuves qui sont mesurables, comme le saut en hauteur, le lancer ou le grimper. Le parcours perd sa finalité initiale de produire et de mesurer une capacité d'adaptation et rejoint le principe

20 Cf. J.-L. Dupuy, « ce système devait permettre de réduire le plus possible les écarts entre les performances 'isolées’ et les performances parcours ». J.-L.Dupuy, "Les parcours contrôlés », Education physique et sport, $\mathrm{n}^{\circ} 55$ (1961) : 15.

21 Ibid., 18. 
Figure 1 : Le parcours-épreuve

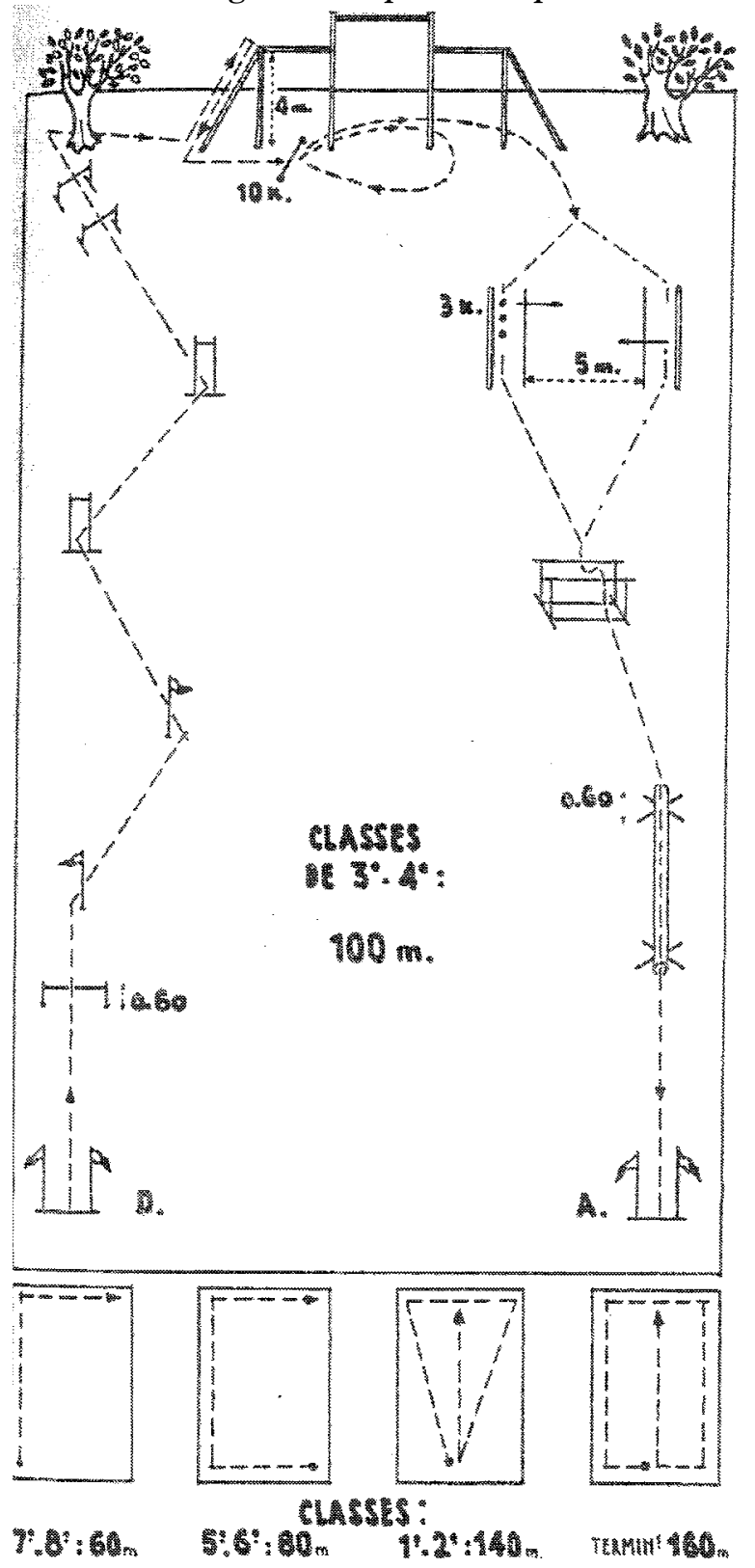

Source : Pierre Parazols, "Groupes de valeur physique et parcours-épreuve », Education physique et sport, $\mathrm{n}^{\circ} 3$ (1950) : 25 . 
des épreuves combinées. Il est constitué de plusieurs épreuves mesurables.

\section{Un complément à l'estimation de la valeur physique lors du parcours : l'évaluation du tempérament par une échelle nominale}

Les échelles nominales permettent de différencier qualitativement deux objets ou deux individus au regard d'une ou plusieurs propriétés communes. Les individus sont ensuite regroupés par catégories sous la même dénomination. Les échelles nominales ne fournissent qu'une information qualitative peu exploitable par la mathématique ou la statistique. Cependant, les parcours sont l'occasion, au tout début des années 50 , de porter des jugements de valeur sous la rubrique générale du tempérament. Ces jugements, que suggère le tableau de Pierre Parazols, permettent de placer les élèves dans des catégories de caractères comme le téméraire, le hardi, le sage. Ces catégories sont elles-mêmes corrélées à des indices de valeur hiérarchisés symbolisés par des lettres. À l'issue de l'épreuve, le professeur interprète certains comportements - tels celui de heurter un obstacle ou de revenir en arrière pour franchir l'obstacle oublié - comme révélant des traits de personnalité. «Le parcours devient un moyen commode d'apprécier, en plus de la valeur physique globale du sujet, les premiers linéaments de sa personnalité ... » ${ }^{22}$.

\section{Les épreuves de composition comme évaluation de la capacité physique}

Une façon de pallier les insuffisances métrologiques du parcours, tout en conservant un regard large sur les capacités de l'élève, est de tenir compte des résultats des compositions trimestrielles. Les compositions sont constituées de deux groupes d'épreuves : les épreuves athlétiques $(60 \mathrm{~m}$, hauteur, grimper, lancer) et gymniques. Les premières représentent les $2 / 3$ de la note, les secondes le $1 / 3$. Le barème utilisé est souvent conçu dans l'établissement ${ }^{23}$. Les résultats

22 Pierre Parazols, " Groupes de valeur physique et parcours-épreuve ", Education physique et sport, $\mathrm{n}^{\circ} 3$ (1950): p.26.

23 " Le choix des épreuves de classement et leur cotation ont été souvent et sont encore remaniés, à la recherche d'une perpétuelle amélioration ». Lucienne Boutin, «Les groupes d'aptitude homogènes au lycée Marie-Curie », Education physique et sport, $\mathrm{n}^{\circ}$ 52 (1960): 11. 
des compositions de l'année précédente permettent de fait de définir des groupes de valeur physique homogène. Seuls les élèves de $6^{\mathrm{e}}$ subissent les tests au début de l'année scolaire.

Le reproche le plus vif adressé à ces outils d'évaluation est leur très grande approximation. Ainsi, Jean Letessier juge-t-il les épreuves très nombreuses, puisqu'il y a des parcours chronométrés (parcours d'obstacle et parcours de sports collectifs), des épreuves gymniques, des épreuves athlétiques, des épreuves de natation, des épreuves « techniques » comportant des exercices de force (les épaulers). Chacune de ces épreuves utilise un référentiel de mesure différent. Tantôt les épreuves sont appréciées, tantôt elles sont mesurées. D'autre part, l'ensemble est converti sur une échelle de vingt points pour fournir une note. Cette conversion est loin de respecter les principes mathématiques et statistiques qui pourraient donner aux notes une équité véritable:

«Mais il [le barème sur 20] présente de graves inconvénients. D'abord celui de la performance-plafond dans chaque épreuve. Ce plafond - si le barème est bien fait - est fixé d'après un certain pourcentage de réussite contrôlé ou supposé dans la population intéressée. Si le pourcentage prévu est faible, donc la performance-plafond élevée, le barème est très étendu et le partage en 20 échelons, trop grossier, discrimine mal. Si le plafond est abaissé, le pourcentage de réussite est plus grand et alors un nombre important de sujets atteint plus ou moins facilement cette limite, ce qui du point de vue sportif, comme du point de vue éducatif, est inadmissible $»^{24}$.

\section{Bilan de l'évolution des instruments d'appréciation de la valeur physique}

On constate qu'en une dizaine d'années, de 1950 à 1961, l'ambition d'une estimation globale de l'élève pour l'établissement de groupes homogènes s'est progressivement amenuisée. Le parti pris d'une mesure objective sur quelques épreuves s'est affirmé. On assiste à une quasi-disparition des échelles nominales très qualitatives qui se référent à des traits du caractère. Le parcours évolue considérablement dans sa nature. L'intention d'inspiration hébertiste

24 Jean Letessier, « Apprécation de la valeur physique », Education physique et sport, $\mathrm{n}^{\circ} 38$ (1958) : 50. 
de faire une «estimation de l'homme complet » n'a pas abouti. La volonté de mesurer, l'exigence d'objectivité, ont contrecarré la finalité intrinsèque de ce dispositif. Le projet explicite de rationalisation de l'éducation physique n'a pu faire aucune concession quand il s'est agi de faire du parcours un outil d'évaluation fiable. Le critère temporel n'est pas suffisamment précis. Il n'exprime qu'une valeur très approximative des capacités qui interviennent dans le déroulement des différentes épreuves. La réalisation du parcours génère de lui-même des déséquilibres qui perturbent l'apparition des différents facteurs de la valeur physique. C'est un instrument qui n'évoque que très grossièrement une valeur générale; au mieux il rend compte de quelques capacités de manière très partielle. Qu'il soit un moyen de développer la valeur physique, on peut le croire. Qu'il soit fait pour estimer la valeur de «l'homme complet», c'est douteux estiment les membres du groupe $\mathrm{n}^{\circ} 5$.

Tout au long de la période, entre 1950 et 1960, les parcours gagneront en précision par des découpages en épreuves barémées puis mesurées. Ces découpages se font pour circonscrire au mieux une seule capacité. La tendance s'oriente vers la différenciation des épreuves, c'est-à-dire paradoxalement vers le type d'épreuves isolées tant décriées à l'origine par les hébertistes du groupe $\mathrm{n}^{\circ} 5^{25}$. Cette dénaturation complète du parcours provoque au tournant des années 60 leur disparition en tant qu'instruments de mesure. Les compositions trismestrielles qui comportaient parfois des parcours ne les retiennent plus. Les parcours ne sont pas satisfaisants. Dans ce sens, Lucienne Boutin écrit :

« La première année (1955), nous avons formé nos groupes à la rentrée sur parcours chronométrés aussi complets que possible et différents suivant la série de classes. Ceci ne nous a pas apporté satisfaction totale (long: 200 élèves environ à faire passer sur un même parcours avec des moyens de chronométrage peu perfectionnés - influence possible de conditions atmosphériques variables - mise en jeu fort insuffisante des éléments coordination, correction, rythme). La deuxième et la troisième année d'expérience, le parcours a cependant été maintenu en début d'année pour les $6^{\text {eme }}$ - abandonné depuis - tandis que

25 Voir Pierre Pierre Parazols, " Groupes de valeur physique et parcours-épreuve », Education physique et sport, $\mathrm{n}^{\circ} 3$ (1950) : 24-26 et Paul Chauchureau, " Le parcours de sport collectif », Education physique et sport, $\mathrm{n}^{\circ} 37,(1957): 22-23$. 
pour les autres classes : classement fait d'après la composition du troisième trimestre de l'année précédente $»^{26}$.

Les problèmes matériels et surtout les problèmes de fiabilité métrologiques ont accentué l'éviction du parcours.

Les compositions, quant à elles, posent des problèmes spécifiques d'étalonnage. D'une part, les barèmes ne sont pas généralisés, chaque établissement possède le sien et, d'autre part, la conversion des performances sur une échelle en vingt points est très imprécise lorsqu'on prétend cerner telle ou telle capacité.

Les praticiens et ceux qui parmi eux sont devenus des théoriciens de la valeur physique et de la mesure ont penché en fin de compte pour l'objectivité. L'estimation de la valeur physique, entendue comme capacité motrice générale, n'est plus poursuivie, de même la capacité globale en sport collectif. La démarche analytique prévaudra. Associée à des situations simples, elle permettra d'identifier une capacité dominante dont on pourra souvent quantifier la performance.

\section{0 : Les éléments de la nouvelle rationalité méthodologique}

L'année 1960 est une date fondamentale car elle traduit un moment de maturité. Les éléments de rationalisation de la pratique enseignante sont, dans l'ensemble, disponibles. Il ne restera plus, par la suite, qu'à les généraliser à la profession. Reprenons ces éléments. L'objet, désormais identifiable est l'ensemble des capacités motrices. Le but de l'enseignement est le perfectionnement de l'élève. Les moyens sont l'ordonnancement des contenus sous forme de programmes et l'individualisation comme méthode d'enseignement. Tout ce système est contrôlé par une évaluation qui est autant un moyen de contrôle des acquis pour l'élève, un moyen d'apprentissage, qu'un moyen de contrôle du rendement de l'enseignant. Ce contrôle se fera en cours d'enseignement par des critères ; l'évaluation terminale, elle, se fera par une épreuve simple et pratique, le pentathlon athlétique ; l'outil de mesure est la table de cotation des performances athlétiques élaborée par Jean Letessier et dont nous ferons état plus loin. Voyons maintenant la genèse de ces différents éléments. Et comme l'objectif fondamental est le perfectionnement de l'élève, la démarche préliminaire consiste à contrôler le niveau de départ de l'élève. Nous commencerons donc par l'outil de mesure. 


\section{La rationalité par la mesure : la nouvelle norme métrologique}

Le projet de mettre en place une pratique d'enseignement contrôlée n'est pas, dans sa réalisation, linéaire et rapide. Les détours sont nombreux, les moyens inégaux. Si la phase de départ, la phase d'identification de la valeur physique, a été accomplie assez vite au début des années 50, les moyens de l'estimation, on vient de le voir, ne sont pas tous concluants. La progression vers des modes de rationalité plus puissants se poursuit puisque ce que l'on vise à terme c'est une épreuve fiable et un outil de mesure, lui aussi, fiable. Ces deux éléments ne seront produits, dans les années 60, que lorsque seront assimilés les moyens intellectuels permettant de les construire. Il faudra donc en passer par la maîtrise d'une culture de la raison, une culture de l'objectivité et de la mesure, il faudra conquérir l'esprit scientifique.

\section{Le climat intellectuel de la période : la psychologie quantitative et la statistique}

Durant cette période qui globalement s'établit sur une vingtaine d'années, la culture de référence du champ de l'éducation physique est essentiellement la psychologie positive et quantitative. La psychologie des aptitudes est associée à un appareillage quantitatif : la statistique. Cette tradition psychotechnique a des précurseurs remarquables en France puisque dès 1905 Alfred Binet et Théodore Simon réalisent la première échelle métrique pour la mesure de l'intelligence afin de reconnaître les élèves aptes à l'enseignement ${ }^{27}$.

Les éducateurs physiques qui, après 1945, réfléchissent sur l'estimation des capacités se réfèreront directement à deux ouvrages des années 1920 se situant dans l'héritage des fondements de Binet. Le premier est L'introduction à la pédagogie quantitative de Ovide Decroly et Raymond Buyse (1929) ${ }^{28}$, le second, Les principes de psychologie appliquée de Henri Wallon $(1930)^{29}$. Ces deux ouvrages se caractérisent par leur option à la fois positiviste et quantitative du traitement des faits psychologiques. Le choix de ces références s'explique assez logiquement par la communauté des thèmes et par le fait que les

27 Alfred Binet, Echelle métrique de l'intelligence (Paris : Société pour l'étude psychologique de l'enfant, 1905).

28 Ovide Decroly, Raymond Buyse, Introduction à la pédagogie quantitative (Bruxelles : Lemartin, 1929).

29 Henri Wallon, Principes de psychologie appliquée (Paris : Armand Colin, 1930). 
auteurs du champ de l'éducation physique avaient dû les connaître en formation initiale dans l'entre-deux-guerres. Des références plus contemporaines sont aussi exploitées, comme celle de Gaston Mialaret, L'éducateur et la méthode des tests, ou celle de J-M Faverge, Introduction aux méthodes statistiques en psychologie appliquée, deux ouvrages parus dans les années $50^{30}$.

Le positivisme qui caractérise cette littérature est fortement induit par «l'esprit» de la mesure. La mesure des capacités ou des aptitudes tend tout naturellement à valoriser une méthodologie de recherche comportementaliste. Le "réaliste » en matière d'éducation, nous disent les tenants belges de la pédagogie scientifique, est celui qui s'intéresse " aux résultats, aux effets, aux conséquences, aux faits : c'est un esprit scientifique $»^{31}$. L'objectivité est donnée par deux éléments : les manifestations comportementales et une échelle de mesure dûment étalonnée. Henri Wallon reprendra ces deux critères fondamentaux pour la méthode de tests :

«Au lieu de supposer derrière les manifestations intellectuelles une sorte de principe autonome, elle [la méthode des tests] a pris pour objet d'étude ces manifestations elles-mêmes. Elle a mesuré, comparé les variations, reconnu les corrélations ou l'indépendance réciproque. Ainsi sont apparus des affinités ou des antagonismes qui font voir l'intelligence en action, et qui en rattachent les manifestations au comportement et au tempérament de chacun. C'est en partant des réalisations extérieures qu'il est possible de distinguer entre les fonctions mentales comme entre les individus $\gg{ }^{32}$.

Ces exigences méthodologiques pour la mesure de l'intelligence seront tout naturellement appliquées pour la mesure des capacités physiques. Le passage inévitable par les réalisations extérieures comme garant de l'objectivité est un des éléments d'un cadre méthodologique qui se positionne contre l'introspection et le mentalisme. L'aptitude ne peut être considérée comme un principe autonome, comme une entité psychologique ou psychomotrice en tant que telle, elle ne peut qu'être perçue en acte. Ce principe a été établi explicitement par le béhaviorisme watsonien dès 1919. Les éducateurs physiques des années 1950 seront aussi sensibles à ce courant par le biais du manuel

30 Gaston Mialaret, L'éducateur et la méthode des tests (Paris : Ed. Scarabée, 1953); Jean-Marie Faverge, Introduction aux méthodes statistiques en psychologie appliquée (Paris : PUF, 1950).

31 Ovide Decroly, Raymond Buyse, op. cit., 18.

32 Henri Wallon, op. cit., 70. 
de Leornard Carmichael sur la psychologie de l'enfant ${ }^{33}$. Cet ouvrage, qui est traduit en français en 1952, fait référence à Thorndike qui sera, dans cette mouvance béhavioriste, un des premiers à utiliser la statistique dans le milieu scolaire.

La statistique est un outil de traitement quantitatif des données. Elle est en partie la réponse au manque d'objectivité dans l'étude des facultés d'un individu. "L'utilisation de la statistique en pédagogie témoigne surtout d'un besoin plus grand de précision et d'objectivité, elle indique une tendance marquée vers l'esprit scientifique en pédagogie $»^{34}$. Faute de pouvoir produire une norme de comparaison à partir d'un seul individu, il convient d'élargir le nombre des sujets possédant tous, à un certain degré, la qualité visée.

La culture de référence intégrera aussi le travail novateur de Georges Canguilhem sur le normal et le pathologique dont la première publication date de $1943^{35}$. Parazols lui emprunte la définition du normal : " un phénomène normal est un phénomène dont l'exploration permanente est possible et dont les caractères mesurés se révèlent identiques à eux-mêmes pour un individu donné, dans des conditions données et à quelques écarts d'amplitude définie près, caractères qui sont identiques d'un individu à l'autre dans des conditions identiques $»^{36}$. Cette définition considère le normal selon le principe d'identité et précise aussi le cadre de la mesure juste, de la reproductibilité du phénomène par la mesure. Mais l'apport essentiel de Canguilhem est ailleurs. Et Parazols l'a souligné aussi : «Il faut encore lever l'équivocité du terme « normal» en ce qu'il représente tout à la fois, un fait et une valeur attribué à ce fait » ${ }^{37}$. La norme est un fait et une valeur. La question que se posent les éducateurs physiques concerne la norme relative aux capacités de l'élève. Puisque la norme est une valeur, quelle sera cette valeur? Il semble que ce que l'on veut évacuer c'est effectivement une valeur idéale et par là hors d'atteinte. On l'a vu, l'idéal de l'athlète complet est relégué. Car il faut

33 Carmichael affirme au début de l’ouvrage ses positions théoriques : « Ce livre est une démonstration claire que dans la psychologie de l'enfant, la période spéculative est révolue définitivement. De nombreuses acquisitions pratiques aussi bien que théoriques ont été le résultat de l'étude empirique de la croissance de l'esprit humain. Il sera clair que pour le lecteur de ces chapitres on est parvenu à formuler des hypothèses concernant de nombreux aspects spécifiques du développement mental. Les hypothèses ont été, en de nombreux cas, éprouvées en laboratoires ou dans ces situations sociales contrôlées et quantifiables. Les conclusions ainsi atteintes sont très différentes des vagues théories verbales de l'ère préscientifique de la psychologie de l'enfant ». Leonard Carmichael L, Manuel de psychologie de l'enfant (Paris : PUF, 1952), XVII.

34 Ovide Decroly, Raymond Buyse, op. cit., 10.

35 Georges Canguilhem, Le normal et le pathologique (Paris : PUF, 1966).

36 Cité par Parazols, Education physique et sport, $\mathrm{n}^{\circ} 22$ (1954) : 11.

37 Ibid.,12. 
que la norme soit rendue par un comportement observable, répétable, exprimé par une classe d'individu, dans des conditions contrôlées. La norme est donnée par la médiane des performances dûment mesurées sur un échantillon significatif et, pour s'approcher de la valeur physique globale, il faut varier les tests. "Un trait humain ne serait pas normal parce que fréquent mais fréquent parce que normal ${ }^{38} . \mathrm{La}$ norme, du point de vue des capacités, est présente derrière la médiane, car la normalité d'un élève qui a la moyenne est non seulement définie biologiquement mais aussi culturellement. La médiane n'est donc pas le reflet d'un individu empirique mais donne un type.

Quant à Canguilhem, sa problématique essentielle est de différencier le normal et l'anormal. Il cherche à définir la valeur de la norme et montrera d'ailleurs que, dans le domaine du vivant, cette valeur n'a rien d'objectif. Et le recours à la définition statistique de l'individu normal ne le satisfait pas plus car « la statistique ne fournit aucun moyen pour décider si l'écart est normal ou anormal ${ }^{39}$. La statistique ne satisfait pas Canguilhem, elle satisfait pourtant les éducateurs physiques. Ceux-ci cherchent à fabriquer un outil de mesure équitable, une table de cotation ; Canguilhem, lui, cherche le fondement objectif de la norme. Deux démarches différentes. Parazols confirmera le bien-fondé statistique de la norme en s'appuyant sur Léonard Carmichael : "Les normes ne sont pas un critère. Ce sont des statistiques servant à la comparaison des données mathématiques, pour éviter l'erreur possible en généralisant des cas isolés. Ce sont des conclusions d'études scientifiques et, comme telles, ce sont des données indispensables ... $»^{40}$.

Mais, le débat de fond sur la définition de la plus grande objectivité possible va plutôt dans le sens de Canguilhem. Car la statistique n'est qu'un moyen d'étude d'une distribution de performances quantifiées. Elle est, pour Parazols, un outil théorique, simplificateur :

«En tant que fait, la norme est une constante déterminée par des moyennes expérimentalement obtenues sur des sujets dont il est important qu'ils aient été en situation directe et concrète d'action sur eux-mêmes. Le modèle, fruit d'une statistique, est une

\footnotetext{
38 Georges Canguilhem, Le normal et le pathologique (Paris : PUF, 1966), 102.

39 Canguilhem, op.cit., 98.

40 Parazols cite en effet Leonard Carmichael dans « L’estimation des capacités physiques », Education physique et sport, $\mathrm{n}^{\circ} 22$ (1954) : 11 .
} 
'construction théorique '; c'est une simplification commode de la réalité $»^{41}$.

Simplificateur, certes, mais utile. En effet, il ne s'agit pas ici de définir la norme objective mais une norme plus objective. Lorsqu'on fait de la moyenne la norme, on gagne en objectivité, à condition aussi que le test, l'épreuve aient été pertinemment établis pour que la capacité visée soit effectivement exprimée, sinon la statistique reste une simple manipulation de nombres, une manipulation vide :

« comme il semble que l'on trouve dans le concept de moyenne un équivalent objectif et scientifiquement valable du concept de normal ou de norme et comme ce concept apparait d'un usage commode, tout au moins dans ce premier stade qui vise à des fins pratiques, nous nous en servirons comme contrôle des données d'un groupe et des données d'un individu à l'intérieur de ce groupe $»^{42}$.

On retiendra de manière synthétique que l'apport de cette psychologie quantitative fut triple. D'une part, l'approche comportementaliste a permis d'extérioriser les capacités individuelles. Et cette prise en compte des manifestations, lors d'une épreuve-test, est incontestablement un pas vers l'objectivité. Insuffisant certes, au regard d'une norme naturaliste. Mais, entre une forme d'introspection très impressive et une conceptualisation rigoureuse, la connaissance par le comportement observable occupe une place honorable sur une échelle de scientificité. L'utilité pratique d'un tel savoir est, en tout cas, indiscutable. D'autre part, la quantification des performances observées permettra de définir la norme par la moyenne. Ce qui permettra non seulement de donner des notes plus justes mais aussi de comparer les élèves. La dernière chose sera l'appropriation de l'outil statistique pour élaborer une table de cotation. C'est ce que va faire Jean Letessier.

\section{Jean Letessier et la quantification de la mesure}

L'impact du travail de Jean Letessier au sein du groupe $\mathrm{n}^{\circ} 5$ sur «l'estimation de la capacité physique » fut considérable. Il est le maître

41 Pierre Parazols, «L'estimation des capacités physiques », Education physique et sport, $\mathrm{n}^{\circ} 22$ (1954) : 12 .

42 Ibid., 12. 
d'œuvre d'une table de cotation des performances athlétiques, dont il deviendra d'ailleurs l'éponyme. Ses travaux commencent dès 1953. Le groupe élabore expérimentalement un barème type pour évaluer les épreuves du professorat et du monitorat d'éducation physique. Le principe d'établissement de la table est statistique. Il s'agit, à partir d'un échantillon significatif, d'établir la courbe de fréquence des performances.

Le premier problème à résoudre est relatif à la distribution des performances qui n'est pas symétrique. Les performances, en tant que variables, ne prennent pas toutes les valeurs entre $-\infty$ et $+\infty \infty^{43}$. Elles n'ont pas de distribution gaussienne ${ }^{44}$ avec un resserrement central autour de la moyenne. "Sur quelle base étalonner les performances de la table ?», s'interroge Jean Letessier ${ }^{45}$. Les écarts de performances concernant une même épreuve ont-ils la même valeur quand on tend vers le record ? La solution consiste à utiliser la fonction logarithmique qui traduit une progression accordant plus de valeur aux performances proches du record.

À partir de ce barème, les fréquences des performances des élèves se distribuent normalement. La distribution est gaussienne et on peut utiliser les indices de dispersion tels la moyenne et l'écart type. Les écarts types sont un indice type d'écart à la moyenne. Si l'on porte de part et d'autre de la moyenne la valeur de l'écart type, la proportion des performances est voisine de $70 \%$. En calculant la moyenne de chacune des six épreuves (100 mètres, 1500 mètres, hauteur, longueur, poids, et grimper), on peut établir les équivalences entre épreuves. De plus, on trouve des équivalences entre les écarts de performances sur des épreuves différentes.

Ces principes mathématiques et statistiques ont permis de dépasser les insuffisances et les incohérences des tables suédoises, finlandaises et portugaises qui emploient un modèle linéaire de distribution des performances. La table donne une conversion des performances en nombre de points, de 10 à 500. Pour faciliter la notation sur 20, Jean Letessier propose un tableau de conversion. La table Letessier est diffusée pour la première fois en 1957. Son concepteur fera pendant presque 10 ans une démonstration très didactique de son usage. En 1961, la seconde édition élargit les références. Enfin, en 1963, paraît une nouvelle édition avec une préface du haut commissaire à la jeunesse et au sport, Maurice

43 Il n’y a pas de mesures négatives pour les performances athlétiques. 44 Dans une distribution gaussienne, les données se distribuent équitablement de part et d'autre de la moyenne.

45 Jean Letessier, op. cit.,10. 
Herzog, qui loue une «table qui permet de chiffrer la progression de chacun depuis le début de l'initiation sportive ${ }^{46}$.

\section{La méthode : l’individualisation par les groupes homogènes}

Toute la démarche de rationalisation de la mesure n'a qu'un but: contrôler l'efficacité de l'enseignement. Selon Parazols, l'enseignement ne peut gagner en efficacité que s'il individualise : "La nécessité de l'individualisation du traitement éducatif s'impose donc, en vertu :

- de la notion même de l'éducation. Pour que l'œuvre éducative soit efficiente, elle doit, en effet, s'adresser non à une collectivité, mais à chaque individu ;

- des différences individuelles constatées ;

- des objectifs assignés à l'éducation physique $»^{47}$.

Faute de pouvoir réellement individualiser l'enseignement, il faut individualiser par défaut, c'est-à-dire faire des groupes homogènes. Mais, pour ce faire, on doit s'opposer à la logique scolaire qui constitue des classes par niveau d'âge, comme si l'âge chronologique et le développement intellectuel étaient systématiquement corrélés. La constitution des groupes physiques homogènes s'avère indispensable parce qu'au même âge la valeur physique des élèves est différente et les écarts sont tels qu'ils hypothèquent l'efficacité d'enseignement ${ }^{48}$. Homogénéité de développement ou homogénéité d'aptitudes ? La question mérite d'être posée, car des élèves qui ont la même morphologie n'ont pas systématiquement le même âge et n'ont peutêtre pas aussi exactement le même niveau d'aptitude.

Le travail en "groupes de valeur physique » est un principe éducatif: «Une véritable éducation suppose de la part de l'éducateur un effort d'individualisation de son enseignement pour l'adapter, le

46 Jean Letessier, « Barème de cotation », Education physique et sport, $\mathrm{n}^{\circ} 67$ (1963), Préface.

47 Pierre Parazols, « Méthode nouvelle ou nouvelles méthodes », Education physique et sport, $\mathrm{n}^{\circ} 44$ (1959) : 18.

48 " Cette prise en considération des différences individuelles ne doit pas faire conclure, pour autant, à l'opportunité d'un traitement purement individuel. Il s'agit de toute façon, de s'adapter aux possibilités de chacun à travers le cadre collectif, de chercher un compromis entre le mode collectif et le mode individuel d'enseignement ». Pierre Parazols, « Les groupes homogènes ", Education physique et sport, $\mathrm{n}^{\circ} 27$ (1955) : 11. 
mettre à la portée de chaque enfant, et, de la part de l'élève, un besoin d'apprendre, un besoin d'être aidé »" ${ }^{49}$. Ce mode pédagogique présente plusieurs avantages. Le premier est «le dépistage et l'amélioration des points faibles $\gg^{50}$. Le second, le développement des aptitudes. D'autre part, les avantages pratiques ne sont pas négligeables : les élèves exécutent plus vite les exercices, les engins n'ont pas à être réglés pour chaque élève, les équipes homogènes progressent plus vite vers les habiletés sportives plus complexes.

Le groupe homogène permet deux autres modalités de groupement propices aux apprentissages : l'équipe et l'atelier. "L'équipe est un petit groupe de six à huit enfants, de structure simple, plus ou moins stable, à l'autonomie relative, dont les buts poursuivis réclament de ses membres une action coordonnée $»^{51}$. Le travail en équipe doit être cadré pour être efficace. Ce cadrage sera mis en place par l'éducateur, un chef d'équipe veillera au respect des consignes. Dans le cas contraire, comme c'est souvent le cas avec les élèves de $\sigma^{\mathrm{e}}$, apparaissent des comportements préjudiciables : l'effort est artificiel, le dégoût de l'activité s'instaure, des conflits apparaissent, des chefs d'équipes peuvent être tyranniques ou indolents. Les principes d'organisation doivent être apportés par l'éducateur qui doit "proposer et répartir les tâches »; le chef d'équipe qui contrôle l'ordre de passage des élèves note sur une fiche le taux de réussite de chacun. La bonne organisation produit de facto une forte motivation ${ }^{52}$.

L'atelier est « un procédé de travail par petits groupes autonomes à des appareils ou sur des emplacements séparés. Généralement, on conserve le système des équipes pour l'appliquer $»^{53}$. Le principe du travail en atelier est assez proche de celui du travail en équipe, l'autonomie relative des élèves les anime tous deux. Cependant le travail par atelier permet à l'éducateur de " réunir les élèves qui présentent un même point faible ou ceux qui sont susceptibles de se perfectionner dans une même technique, un même style, et de leur permettre ainsi de poursuivre en commun un but déterminé ${ }^{54}$. L'équipe et l'atelier sont deux modes assez proches du groupe homogène, la seule différence est que l'atelier accentue encore plus la différenciation des élèves. L'équipe est encore un groupe homogène

49 Pierre Parazols, "Groupes et ateliers », Education physique et sport, $\mathrm{n}^{\circ} 8$ (1951) : 12 .

50 Ibid., 24.

51 Pierre Parazols, "Le groupe et l'équipe ", Education physique et sport, $\mathrm{n}^{\circ} 6$ (1951) : 12.

52 « Le groupe organisé est plus fortement motivé dans la coopération ». Pierre Parazols, op. cit., 12.

53 Pierre Parazols, ibid., 12.

54 Ibid., 13. 
par niveau de capacités, l'atelier est un groupe homogène par niveau de compétences.

Mais les griefs à l'encontre d'un tel procédé sont nombreux. L'organisation matérielle, la mise en pratique sont lourdes, car il faut que toutes les classes d'un même niveau soient alignées. La constitution des groupes homogènes, différents du groupe classe initial, fait que «l'esprit de classe est rompu» ${ }^{55}$. Les groupes Education physique et sport, effectivement, n'associent plus les élèves d'une même classe, mais certains, seulement. Ce qui pourrait générer des clans et troubler l'harmonie psychologique de la classe. Ensuite, le groupe homogène, de par son principe même, nuirait à l'émulation. Comme les élèves ont tous le même niveau, la motivation par la différence des compétences serait quasi- inexistante. Enfin se pose le problème du contrôle des présences. Les listes d'appel sont établies par classe et le professeur d'éducation physique se doit d'établir de nouvelles listes ; d'où une charge de travail supplémentaire.

À chacun de ces griefs, les promoteurs du projet répondront et seront appuyés en cela par l'autorité institutionnelle. Le 25 juin 1959, une circulaire émanant de la Direction générale de l'organisation et des programmes scolaires, cosignée par Maurice Herzog, Haut commissaire, et Lucien Paye, Directeur Général de l'Organisation et des Programme scolaires du ministère de l'Éducation nationale, précise que « De nombreuses expériences réalisées sur plusieurs années scolaires, et qui viennent d'être minutieusement analysées, ont mis en évidence les avantages indiscutables retirés d'une organisation de l'éducation physique et sportive basée, non plus sur l'existence des classes ou divisions, mais sur la constitution de groupes d'aptitude physique homogènes ». La circulaire stipule aussi que la constitution des groupes homogènes n'est pas obligatoire, mais elle est recommandée «avec insistance » dans « tous les cas où une même classe comporte plusieurs divisions $»^{56}$.

55 Lucienne Boutin, op.cit., 12.

56 Direction générale de l'organisation et des programmes scolaires. Circulaire du 25 juin 1959. À noter que le texte de la circulaire est repris comme exerg ue de l'article de Lucienne Boutin, " Les groupes d'aptitudes physiques homogènes au lycée MarieCurie », Education physique et sport, $\mathrm{n}^{\circ} 52$ (1960) : 10. 


\section{Le choix d'une seule épreuve normalisée : le pentathlon athlétique}

«Comment, à travers des épreuves simples, est-il possible, avec une assez bonne approximation, d'apprécier la valeur physique d'un sujet, de juger de ses progrès, de le situer par rapport à son groupe? $\gg{ }^{57}$. Telle est la question que pose Jean Letessier à la fin des années 1950. Les instruments du contrôle - épreuves et table - sont désormais établis. Les épreuves sont limitées à cinq et l'instrument de mesure - la table - présente toutes les garanties théoriques de fiabilité. L'intention des éducateurs du groupe $\mathrm{n}^{\circ} 5$ est de généraliser le pentathlon et l'usage de la table à toutes les instances éducatives et sportives qui évaluent la valeur physique : «Un projet de pentathlon athlétique étudié et proposé par le groupe d'étude répond à ces conditions. Son application généralisée introduirait dans l'enseignement de l'éducation physique un élément cohérent, unifié, généralisé et simplificateur $»^{58}$. La première de ces instances est le BSP, le brevet sportif populaire instauré par Léo Lagrange en 1937 ; la seconde est le triathlon de l'Office du sport scolaire et universitaire (OSSU), et enfin la troisième, les examens scolaires du BEPC ${ }^{59}$ et du baccalauréat.

Le brevet sportif populaire est une épreuve de masse qui a pour but d'évaluer la capacité physique de tous les Français. Il comporte pour les hommes un lancer de poids ( $7 \mathrm{~kg} 257$ ), un saut en hauteur avec élan, une course de $100 \mathrm{~m}$ plat, un grimper de corde (bras seuls) et une course de $1000 \mathrm{~m}$ plat; pour les femmes : un lancer de poids (4 kg), un saut en hauteur avec élan, une course de $60 \mathrm{~m}$ plat et un grimper à la corde (bras et jambes); la natation (25 m nage libre) est facultative. Les performances pour l'obtention du brevet sont basses (1,20 m en hauteur ou 15 secondes au $100 \mathrm{~m}$ pour les hommes) et correspondent à la cote zéro, l'état de débrouillardise des épreuves du Code de la force de G. Hébert.

L'OSSU organise chaque année un triathlon, une épreuve de masse pour les élèves, qui a pour but de dégager une "élite athlétique ». Ce triathlon comprend : la vitesse, le saut en hauteur et le lancer de poids. Les modalités de l'épreuve obligatoire d'éducation physique au baccalauréat, stipulées par l'arrêté du 5 octobre 1959, comprennent quatre épreuves : une épreuve gymnique, une épreuve

57 Jean Letessier, « Pentathlon athlétique et appréciation de la valeur physique », Education physique et sport, $\mathrm{n}^{\circ} 44$ (1959) : 19.

58 Pierre Parazols, Parazols, " Méthode nouvelle ou nouvelle méthode ? », Education physique et sport, $\mathrm{n}^{\circ} 44$ (1959) : 16.

59 Brevet Elémentaire du Premier Cycle des collèges. 
de grimper libre, une épreuve d'athlétisme, tirée au sort parmi la hauteur, la course de vitesse et le lancer de poids, et une épreuve choisie par le candidat parmi les épreuves d'athlétisme pré-citées. S'y ajoutent pour les garçons une course de résistance, pour les filles un lancer du poids ou un lancer d'adresse et pour les deux sexes une épreuve chronométrée de nage libre. Parmi ces épreuves, seule l'épreuve gymnique n'est pas barèmée; elle est sensée tempérer la seule estimation des aptitudes innées par une prise en compte du progrès.

Le BSP, l'OSSU, le BEPC et le baccalauréat impliquent des épreuves différentes et des barèmes différents. Jean Letessier et les membres du groupe veulent dépasser cette trop grande disparité et proposer dans un premier temps une uniformisation par un barème unique. La table Letessier deviendrait le seul référent des différentes épreuves scolaires et non scolaires. À terme, l'intention est de réduire ces épreuves au seul pentathlon athlétique qui se réaliserait à la fin de l'année scolaire. Le «carnet scolaire d'éducation physique», dont une version a été testée concrètement, présente d'ailleurs comme épreuves type d'éducation physique "les cinq épreuves du pentathlon ${ }^{60}$.

\section{Rendement, contrôle, normalisation : le bilan}

«Qu'attend l'Inspecteur de la substance même de la leçon ? Qu'on obtienne du progrès; telle est la règle d'or dans toute tentative éducative $»^{61}$. Roger Marchand n'exprime pas là uniquement le point de vue de celui qui est désigné par l'institution pour contrôler, c'est-àdire étymologiquement assurer le contre-rôle des éducateurs physiques ; il ne fait que traduire le souci d'une corporation qui se veut légitimement productrice d'effets. Car le progrès, c'est « une différence positive entre le début et la fin de la leçon ${ }^{62}$. Légitimité, car que serait un enseignement qui ne transmettrait rien, ou qui serait incapable de montrer qu'il a transmis quelque chose. Éduquer, c'est transmettre des normes et des savoirs; aucune société n'y échappe. Et dans une société démocratique, éduquer, c'est transmettre des normes et des savoirs spécifiques en vue de l'émancipation de la génération montante qui aura à trouver son propre mode d'adaptation à un monde à venir. La responsabilité démocratique s'exerce aussi en évaluant les compétences de chacun, en ayant donné à chacun

60 Ibid., 25.

61 Roger Marchand, «L'inspecteur pédagogique et la leçon d'éducation physique et sportive », Education physique et sport, n ${ }^{\circ} 70$ (1964) : 10.

62 Ibid., 9. 
l'occasion d'exprimer au mieux son potentiel. C'est le souci de Roger Marchand qui demande : «Que va gagner l'enfant, la classe ? l'enfant, la classe vont-ils gagner le maximum possible? ${ }^{63}$. Tout comme les autres professeurs, le professeur d'éducation physique se doit de transmettre un savoir. Il se doit aussi, c'est sa déontologie, d'être à même de contrôler le savoir qu'il transmet. Cette quête de l'efficacité doit donc, désormais, devenir la norme. La norme de toute une profession.

Tout comme on généralise les unités de mesure et les monnaies pour faciliter le commerce, les éducateurs physiques vont opter pour le même instrument, la même table pour qu'il y ait commune mesure, pour que les notes aient pour tous la même signification, quel que soit le lieu et quel que soit l'établissement. Souci premier de mettre de l'ordre, pour que l'enseignement soit efficace avant tout pour l'élève. Dans ce sens, Georges Colo précise :

«Et pourtant, il est un remède bien simple : normaliser ! Faire pratiquer l'éducation physique sans mesurer les aptitudes et contrôler ces résultats, c'est travailler dans la nuit. Mais si pour contrôler ces résultats, il est imposé autant de barèmes de cotation et autant d'épreuves différentes qu'il est proposé d'examens à préparer et subir, c'est rendre la nuit plus noire encore. Un éducateur physique répugne à travailler dans le désordre. Le mètre et le chronomètre le forgent net, loyal et objectif. Education Physique aux examens ? Crise de l'athlétisme ? Prestige légitime d'une corporation gagnant son pain à la sueur de son front? Désir d'être pris au sérieux ? D'être entendus ? - Entendons nous d'abord pour être entendus $\gg{ }^{64}$.

Revendication de la norme qui ne peut être pensée comme arbitraire et qui relève plutôt du bon sens, du bon sens pratique : "Conclusion en forme de plaidoyer pour la normalisation en éducation physique ... Il est de bon sens dans une telle situation, de convenir d'un certain nombre de règles, même imparfaites, mais connues de tous et appliquées par tous. De toute évidence, il en résulte un gain d'économie et d'efficacité, entre autres, et la satisfaction de tous ${ }^{65}$. Normalisation, non seulement de l'éducation physique scolaire, mais aussi des sphères sociales de la pratique

63 Ibid., 10.

64 Georges Colo, « Normalisation », Education physique et sport, $\mathrm{n}^{\circ} 28$ (1955) : 44.

65 Pierre Parazols, « L'estimation des capacités physiques ", Education physique et sport, $\mathrm{n}^{\circ} 22(1954): 13$. 
sportive. Car le pentathlon est l'essence même du sport, il peut devenir le moyen d'évaluation de la valeur physique de tous les Français. Ainsi tout le domaine de la pratique sportive aurait le même étalon de mesure. Généralisation du référent à la nation toute entière : «Le projet de pentathlon athlétique ... comblerait une lacune regrettable dans ce domaine du contrôle des résultats et ouvrirait des perspectives favorables sur les problèmes précités : épreuves, classements ... et sur les liaisons entre l'éducation physique et le sport scolaire, entre le sport scolaire et le sport civil $»^{66}$. Faut-il voir là une collusion entre l'éducation physique et le sport ? Il est indéniable que les membres du groupe d'étude $n^{\circ} 5$ sont des partisans du sport. Et si certains, parmi eux, conservent un ancrage hébertiste, et restent des adeptes des parcours d'obstacle, la plupart seront gagnés, pendant la décennie 50, par l'esprit sportif. Tout le travail de Jean Letessier porte exclusivement sur des «performances sportives » et contre les dérives possibles de la compétition. Il est très clair : « Le sport qui se traduit en performances numériques sera-t-il victime de cette périlleuse attraction des ' plafonds'? Non, s'il réussit à s'implanter profondément dans la vie des jeunes, en particulier dans le milieu scolaire où son rôle n'est pas d'atteindre des sommets mais de susciter une saine émulation dans des compétitions accessibles à tous ${ }^{67}$.

La pédagogie du rendement servira à promouvoir la sportivisation de l'éducation physique. Elle fera le jeu de Maurice Herzog contre la gymnastique construite et même contre les tenants d'un sport éducatif. Toutefois, cette quête d'efficacité traduit une attitude nouvelle, car la corporation de l'éducation physique n'avait, peut-être jamais, été interpellée par la notion de progrès, de progrès démontré tout au moins. Qu'un professeur de sciences naturelles, de langue ou de mathématique contrôle les acquis de ses élèves sous forme d'interrogation écrite, cela a toujours semblé normal. Qu'il y eut des compositions d'éducation physique en fin de trimestre, cela aussi était considéré comme normal, non au sens de l'obligation de produire du progrès, c'est-à-dire de l'apprentissage, mais plutôt au nom d'une ritualisation du temps scolaire. Car, on peut effectivement faire faire des compositions trimestrielles sans que, pour autant, le progrès de l'élève puisse être établi. L'actualisation de compétences n'a jamais signifié leur récente acquisition. Le spectre de l'inutilité, de

66 Pierre Parazols, « Méthode nouvelle ou nouvelle méthode ? », Education physique et sport, $\mathrm{n}^{\circ} 44$ (1959): 16 .

67 Jean Letessier, "Table générale de cotation des performances athlétiques et barèmes pour les examens sportifs », Education physique et sport, ${ }^{\circ}$ 79, (1966) : 18. 
l'inefficacité, de la déconsidération de l'éducation physique est bien présent en arrière-plan.

C'est bien la transmission de véritables contenus d'enseignement qu'il s'agit de mettre en place désormais. La chose n'est pas facile. Car si l'on a choisi le développement des capacités physiques, il faudra démontrer qu'elles sont susceptibles d'un développement réel en cours d'éducation physique, autrement dit que le cours offre les conditions de leur développement. Quelles sont-elles ? Il faut certes rationaliser la mesure des capacités, la table Letessier, globalement, le permet; mais il faut ensuite que les méthodes soient optimalisées, que le temps scolaire d'entraînement soit suffisant et surtout que les conditions matérielles de la pratique soient bonnes. Dans ce sens, Jean Pinturault dresse un bilan rapide et précis :

«Les leçons d'Education physique doivent constituer pour nos scolaires des modèles, des exemples à inclure dans le contexte d'une vie physique : deux heures par semaine ou plutôt une centaine de minutes, introduites au hasard des horaires d'un établissement, un plein air de cent vingt à cent cinquante minutes, ne semblent pas fournir un menu suffisamment cohérent pour améliorer ou perfectionner le scolaire $»^{68}$.

Un dernier problème est lié à la nature même des pratiques qui supportent l'enseignement ${ }^{69}$. Quant à choisir le sport, peut-on le réduire à un pentathlon en occultant les sports collectifs ou les sports duels ? Et puis, toutes les pratiques sportives ne reposent pas exclusivement sur des capacités athlétiques, elles font intervenir aussi des habiletés motrices qui s'apprennent par apprentissage. Il faut le craindre, toutes ces conditions ne seront pas remplies. La plus fondamentale, celle qui concerne les installations, est encore, dans les années 60, largement irrésolue. Qu'apprend-on réellement lors des séances de plein air, surtout quand les intempéries les rendent impossibles? Les Instructions de 1959 évoquent cette situation, relativement fréquente: «Par mauvais temps caractérisé, et du fait que de nombreux établissements ne disposent pas de gymnase ou autre

68 Jean Pinturault, «Leçons d'éducation physique », Education physique et sport, $\mathrm{n}^{\circ} 52$ (1960) : 19.

69 Constatant "l'insuffisance des épreuves cotées athlétiques », Le Boulch souligne : «Lorsqu'un élève a progressé dans une de ces épreuves d’une année à l'autre, la cause du progrès est très difficile à déceler. Est-ce parce que cet élève a grandi, est devenu plus lourd, plus fort, plus adroit, ou bien est-ce parce qu'on lui a enseigné une «recette » particulière lui conférant un nouveau savoir-faire. » Jean Le Boulch, « Esquisse d'une méthode rationnelle et expérimentale d'éducation physique », Education physique et sport, $\mathrm{n}^{\circ} 57$ (1965) : 36. 
surface couverte, l'éducateur doit s'attacher à exploiter une situation qui, si elle est moins favorable à l'enseignement des disciplines corporelles, permet néanmoins des activités de remplacement non négligeables $»^{70}$. La responsabilité de la transmission d'un contenu d'enseignement exclusif doit aussi tenir compte de deux autres facteurs : la croissance qui fait que chaque élève voit ses propres capacités augmenter naturellement et, d'autre part, la pratique concurrentielle extra-scolaire qui agit aussi sur les capacités.

Le bilan d'un acquis moteur effectif à mettre sur le seul compte d'une éducation physique n'est pas réalisable. D'où le postulat de Letessier, selon lequel, l'effet de l'enseignement - entraînement - est équivalent pour tous les élèves. Une façon d'éluder le problème.

\section{Conclusion : Quelles normes de rationalité pour la pédagogie du rendement?}

Si l'on considère que le rationnel est ce qui obéit à des règles, on distinguera deux types de rationalité : une rationalité du connaître, rationalité théorique, et une rationalité de l'action, rationalité pratique.

La rationalité du connaître a été donnée en partie par la capacité qu'ont les tests de donner un aperçu empirique de la manifestation d'une capacité. Et ce cheminement vers le donné empirique est appréciable, il est préférable en tout cas à une simple postulation spéculative ou nominale. Les problèmes ne sont pas tous levés, car il n'y a pas de capacités complètement identifiables et absolument différentiables comme le sont les phénomènes homogènes de la chimie ou de la biologie. Une capacité ne peut qu'être révélée. Gain de rationalité parce que gain d'objectivité. A cela, il faut ajouter l'usage de la statistique qui donne aussi un gradient d'objectivité par le traitement des grands nombres, c'est-à-dire par la multiplication des cas singuliers. Letessier, qui étalonne sa table en veillant à la normalité des distributions, pourra établir des comparaisons entre les différentes épreuves. Ces deux plans aboutissent à une mesure fiable, à un outil de classement efficace, et l'exigence pratique s'en satisfait grandement.

La rationalité pratique présente une autre nature. Elle est relative à la saisie concrète du monde, du monde humain surtout. Elle est aussi associée à un but, un résultat, un succès escompté. Dans ce sens le mode de rationalité de la pédagogie du rendement est une rationalité de

70 Instructions du 20 juin 1959. Ces moyens " non corporels » sont : la lecture sportive, les films et commentaires, et de nombreux thèmes théoriques : l'histoire du sport, les grands explorateurs, la place du sport dans la nation, le secourisme etc. 
l'action selon un mode technique. L'efficacité et la vérifiabilité sont, ici, les critères de la raison. Car, si les éducateurs font la démonstration que les élèves se perfectionnent, ils auront agi selon ce mode rationnel. L'atteinte du but concret, ici les progrès de l'élève, est relative au moyen, au respect de l'ordre dans la progression. D'où l'importance du cadre global et réglé de la pratique - l'évaluation préalable, la définition des objectifs, le programme, la production des groupes homogènes, l'individualisation, la progression dans l'apprentissage par les exercices-critères, l'évaluation terminale par le pentathlon -, autant d'éléments qui sont les étapes d'une pédagogie soucieuse d'atteindre son but. Rationalité faible, certes, qui n'est pas celle du connaitre, mais qui comporte ses propres principes, son propre ordonnancement, sa propre logique pratique.

La pédagogie du rendement est une méthode, dans son sens le plus instrumental, mais elle reste gouvernée par une norme, une valeur, une conception de l'homme : celle d'éduquer physiquement un élève en développant son potentiel moteur, ses capacités. La finalité est première, la raison pratique qui intègre la méthode est le moyen de l'atteindre.

Cette raison pratique est méthodologique, elle est surtout organisationnelle. L'organisation prévoit et prépare les conditions de l'action en vue de son efficacité. L'organisation de la pédagogie du rendement doit être anticipée pour « libérer l'éducateur de certaines contraintes, lui donnant ainsi la possibilité de mieux se consacrer à son enseignement» pour permettre « de la part des professeurs, une action des plus fructueuses par une répartition rationnelle des horaires et l'adhésion à des règles de travail communes ${ }^{71}$. L'organisation présente trois moments : la répartition des heures de travail de manière que " chaque série de classes de même niveau se présente aux mêmes heures ", ce qui permet de constituer les "groupeséquipes-ateliers »; la répartition des lieux de travail qui se base sur un « roulement dans le temps sur un cycle de six semaines» et, enfin, la répartition des moyens de travail qui comprennent les méthodes d'organisation du travail personnel de l'éducateur physique, le plan d'aménagement des salles et terrains et le programme de travail.

La pédagogie du rendement possède bien une double rationalité, théorique et pratique, mais en tant qu'instrument d'intervention sur le réel a-t-elle pu parvenir à ses fins ? Les expériences pratiques relatées, celles des concepteurs et des praticiens, tendent à le confirmer. Toutefois, des tensions demeurent qui ont fait le jeu de

71 Pierre Parazols, "L'utilisation des installations », Education physique et sport, $\mathrm{n}^{\circ} 13(1952): 34$. 
toutes les formes d'inertie et de conservatisme. Tensions entre un outil d'évaluation - d'estimation - qui se veut à la fois pratique et précis. Pratique, parce que maniable et fiable; précis, parce qu'il est sensé donner une image globale de la valeur physique. Mais les seules épreuves du pentathlon donnent-elles une bonne appréciation de la valeur physique? Qu'en est-il de tous les registres techniques des pratiques? Sont-ils inévaluables ou bien ne sont-ils pas importants?

Tensions entre la démonstration d'efficacité d'un enseignement et l'incapacité qu'il a à la produire. Chercher à perfectionner, à viser un progrès tout en ignorant ce qui est susceptible d'être réellement appris en cours d'éducation physique. Sachant qu'il n'est peut-être pas possible de développer les capacités motrices dans les conditions réelles d'enseignement. Tensions, aussi, entre une exigence de rationalité de la pratique, dont la composante organisationnelle et la dotation matérielle sont le point d'orgue et les conditions communes de la pratique d'enseignement qui sont souvent indigentes. Tensions, enfin, entre la revendication d'un statut disciplinaire analogue aux autres matières scolaires et le maintien d'une place à part, d'une place originale et spécifique. Car la définition du statut de la discipline influe aussi sur l'image sociale qu'elle peut avoir. La responsabilité prend désormais un sens. Le devoir de transmission obéit à des critères moraux et sociaux au nom desquels l'éducateur physique doit rendre compte de ses actes. Il en va du crédit moral et social d'une profession.

La scolarisation de l'éducation physique est accomplie certes, mais ne reste-t-elle pas formelle? Suffit-il d'être une matière obligatoire, avec des objectifs, une individualisation, des compositions de fin de trimestre pour pouvoir dire qu'on a enseigné véritablement, que les élèves ont gagné le maximum possible? Qu'ont-ils gagné en vérité? Tous les enjeux de la discipline peuvent se réduire à cette simple question. 